\title{
Captando la atención del alumnado en el estudio de Ciencia de los Materiales a través de un cambio metodológico
}

\section{Capturing the attention of students in the study of Materials Science through a methodological change}

EVA MARÍA PÉREZ SORIANO

ORCID: https://orcid.org/0000-0003-0658-8098

Universidad de Sevilla

Departamento de Ingeniería y Ciencia

de los Materiales y del Transporte

evamps@us.es

Fecha de recepción: 01-06-2019.

Fecha de aceptación: 14-06-2019.

DOI: http://dx.doi.org/10.12795/9788447221912.012

Pp.: 288-312 
La Ciencia de los Materiales es una rama común de conocimiento a todas las ingenierías que no suele tener el peso que merece. No se transmite a los alumnos la importancia que tendrá en el desarrollo de su vida profesional. Todo está hecho de un material que debe cumplir con unas especificaciones y, sin eso, el desarrollo de productos y procesos carece de sentido. Se han desarrollado cambios en la metodología docente, basados en el enfoque de estudios de casos para enseñar a comprender y evaluar las distintas dimensiones y desafíos globales. De esta forma los alumnos participan en grupos para llegar a resolver los problemas, sin necesidad de explicaciones previas magistrales, sino con pequeñas preguntas clave que les van abriendo camino hasta su resolución completa. Con el cambio metodológico ha habido un cambio de actitud positivo por parte del alumnado, involucrándose muy activamente en su proceso de aprendizaje.

Palabras clave: Fundamentos de Ciencia de los Materiales, Grado en Ingeniería Química, docencia universitaria, experimentación docente universitaria.

\section{Abstract}

Materials Science is a common branch of knowledge to every engineering are, but it does not usually have the place that deserves. The importance in the development of their professional life is not transmitted to the students. Everything is made of a material that must meet specifications and, without that, the development of products and processes is meaningless. Changes have been developed in the teaching methodology, based on the case study approach to teach to understand and evaluate the different dimensions and global challenges. In this way, students participate in groups to solve problems, without the need for prior masterful explanations, but with small key questions that will open the way to their complete resolution. With the methodological change, there has been a positive change of attitude on the part of the students, getting involved very actively in their learning process.

Key words: Materials Science Basis, Chemical Engineering Degree, university lecturing, university teaching experimentation. 


\section{Descripción del contexto}

Esta propuesta educativa de cambio metodológico y actitudinal del profesor se ha aplicado durante el segundo cuatrimestre en dos asignaturas: Fundamentos de Ciencia de los Materiales, de 2 o del Grado de Ingeniería Química, y en Ciencia y Resistencia de Materiales, de 30 del Grado en Ingeniería de la Salud. En ambas asignaturas se cuenta con unos 80 matriculados aproximadamente. En el caso de Fundamentos de Ciencia de los Materiales, ya desde el inicio del cuatrimestre se parte con la mitad del alumnado en las clases, reduciéndose el número hasta unos 15 al final del periodo lectivo. Para la asignatura Ciencia y Resistencia de Materiales, la asistencia de los alumnos es más estable, partiéndose de unos 60 y disminuyendo a 40 durante las clases.

Aunque esta última asignatura es compartida al 50\% con el departamento de Resistencia de los Materiales, la responsabilidad de mi parte recae únicamente en mí, teniendo libertad para elegir los conceptos a impartir y la forma de darlos. Para Fundamentos de Ciencia de los Materiales, la libertad es absoluta, puesto que sólo hay un grupo y soy la coordinadora de la asignatura.

En ambos casos se cuenta con aulas de bancas fijas, sin posibilidad de movilidad para los trabajos en grupo; eso limita las interacciones entre los alumnos, teniéndose que establecer los grupos de trabajo de entre 2 y 4 personas.

Ocurre además que los alumnos del Grado en Ingeniería de la Salud no demuestran a priori demasiado interés por la asignatura, quizás por su carácter excesivamente técnico.

En el caso de los estudiantes del Grado de Ingeniería Química, a estas alturas de curso están algo desconectados porque se encuentran en plena época de parciales. 
Se distinguen dos tipologías de alumnos en clase:

- Los que llevan al día la asignatura y participan bastante en cuanto se les motiva a ello.

- Los que siguen viniendo por no dejar la asignatura, pero se encuentran perdidos y no sólo no participan, sino que se niegan incluso cuando se les intenta obligar a que lo hagan.

Se diseña un ciclo de mejora a desarrollar en el aula (CIMA), para que sea lo más interactivo posible, con la intención de reincorporar a la dinámica de clase a los más rezagados y menos participativos.

\section{Diseño previo del ciclo de mejora en el aula}

Las asignaturas se dividen en tres grandes bloques con el siguiente alcance, en función de la escala de observación: atómico, microscópico y macroscópico. El tema por impartir corresponde al último del bloque II, y se llama "Diagramas de Fases". En él, tienen que aprender a dibujar un diagrama de fases, pero, sobre todo, saber interpretarlo.

En primer lugar, se hizo un estudio de los contenidos a impartir, seleccionándolos por orden de importancia. Para llevar a cabo esta metodología, se requiere de mayor disponibilidad de tiempo frente a lo homólogo con una clase magistral. Habrá que clasificar y acotar aquellos conceptos que puedan tener cabida dentro de la franja lectiva, y aquellos a impartir mediante referencias a apuntes y/o fuentes bibliográficas. En la figura 1, se presenta el mapa de contenidos correspondiente a dicho tema.

Desde la pregunta origen, situada en la esquina izquierda superior, salen dos ramas. En primer lugar, debe seguirse el camino inferior, en el que se explican las condiciones básicas en la interpretación de diagramas, siguiendo condiciones de equilibrio. Se exponen tanto los 
conceptos básicos, como los avanzados necesarios para poder dibujar un diagrama.

A continuación, se tomaría el segundo camino desde la pregunta origen hacia la derecha, donde se estudian las modificaciones causadas por un enfriamiento de las aleaciones fuera de las condiciones de equilibrio; es decir, con un enfriamiento más rápido. Se estudian los distintos casos que se pueden presentar, y se razona cómo van a deformarse los diagramas bajo estas condiciones.

Jornadas de Formación e Innovación Docente del Profesorado | № 2 (2019) Esta obra se distribuye con la licencia Creative Commons 


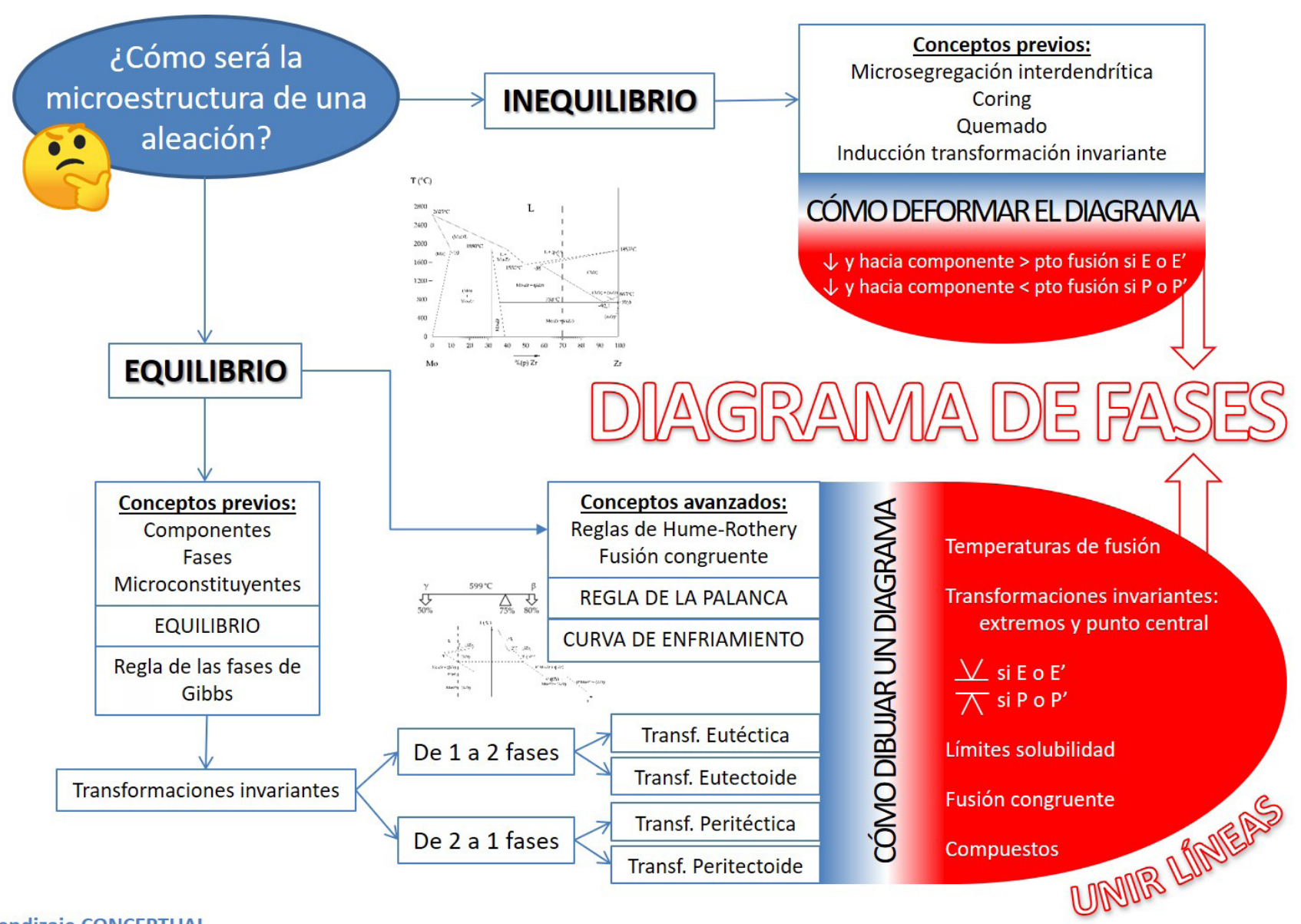

Aprendizaje CONCEPTUAL

Aprendizaje PROCEDIMENTAL

Figura 1. Mapa de contenidos del tema de diagramas de equilibrio

Jornadas de Formación e Innovación Docente del Profesorado | № 2 (2019)

(C) Esta obra se distribuye con la licencia Creative Commons

(c) $)()_{\mathrm{EY}} \mathrm{NC}$ Reconocimiento-NoComercial-SinObraDerivada

Internacional (CC BY-NC-ND 4.0.) 
Al ser un tema de carácter claramente práctico, se desarrollan primero los contenidos conceptuales para poder entender qué se hará después en los contenidos procedimentales, que son la base del tema. Los contenidos actitudinales respecto al ahorro energético o al impacto medioambiental respecto a la selección de materiales, no aparecen en este mapa de contenidos porque son conceptos que pertenecen a futuras asignaturas. Pero debido a su extrema importancia, se introducen dichos conceptos a los alumnos de palabra, para que no pierdan esa perspectiva, aunque no la estudien de lleno aún.

Se presenta en la figura 2 el modelo metodológico deseable que, gracias a este CIMA, se convierte en posible.

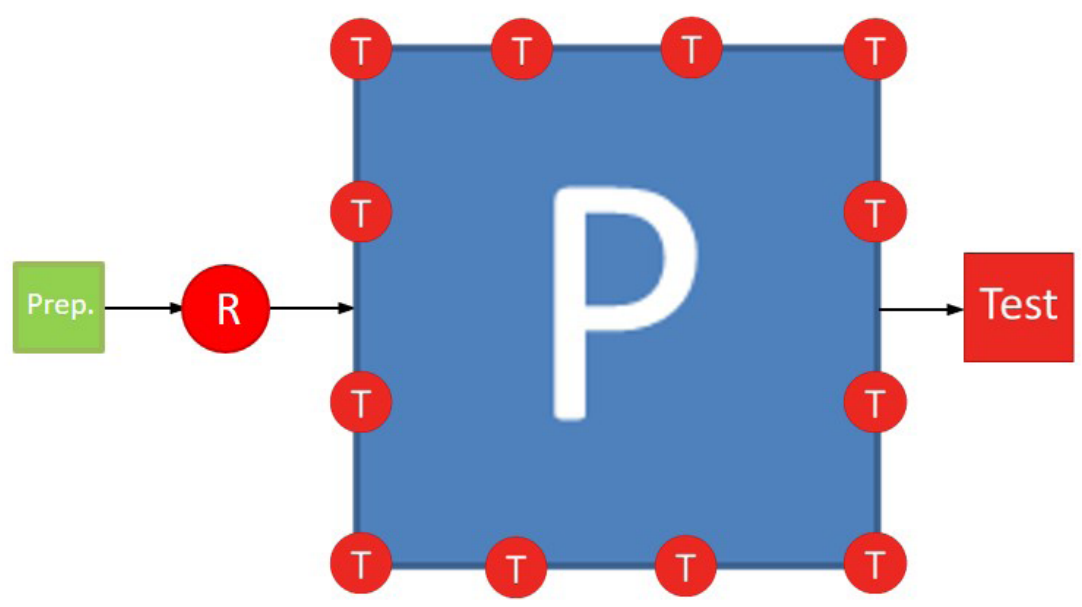

Figura 2. Modelo metodológico deseable/posible

El modelo está basado en la resolución de un problema global. De esta forma, los alumnos se involucran por grupos de unas 6 personas, hasta llegar a resolverlo, sin necesidad de explicaciones previas magistrales, sino con pequeñas preguntas clave que les van abriendo puerta hacia la resolución completa del mismo. A continuación, se presenta la secuencia programada inicial de actividades en este CIMA. En la tabla 1 se incluye la información 
referente a las tareas. La duración total programada para este CIMA fue de 7,5 horas.

Tabla 1. Diseño de la secuencia de actividades

$\begin{array}{ll}\text { Tarea } & \text { Descripción } \\ \begin{array}{ll}\text { 1. Preparación de la } \\ \text { clase (5 } \mathbf{~ m i n )}\end{array} & \begin{array}{l}\text { Presentación de la nueva forma } \\ \text { de dar este tema; } 5 \text { sesiones en } \\ \text { total. }\end{array}\end{array}$

2. Cuestionario inicial (20 min) grupos (5 min)

4. Problema: entrega enunciado (5 min)

Se marca un límite de entre 3 y

3. Formación de 5 miembros, se recomiendan 4 personas por grupo.

No podrá haber más de un repe-

tidor por grupo.

Tiempo para leer el problema y procesarlo. Se puede consultar

cualquier tipo de fuente.

Pregunta 1:

Se darán cuenta que prácti-

camente no tienen por dónde empezar.

\section{Contenidos}

Cómo dibujar

un diagrama

de equilibrio

Componen-

tes-Fases-Mi-

5. Píldora informativa (5 min)

croconsti-

tu-yentes

Transformacio-

nes invariantes

6. Problema: finali-

Pregunta 2:

zar parte 1, pensar

sobre pregunta 2

(20 min)

Análisis de fa-

ses completo,

cómo hacerlo

\section{Píldora informa- tiva (5 min)}

Regla de la

palanca

Análisis de

fase: 5 ítems

\section{Problema: fina-} lizar parte 2, pensar sobre pregunta 3 (20 min)

Pregunta 3:

Diagrama T-t

Jornadas de Formación e Innovación Docente del Profesorado | № 2 (2019) Esta obra se distribuye con la licencia Creative Commons Reconocimiento-NoComercial-SinObraDerivada Internacional (CC BY-NC-ND 4.0.) 
9. Resumen concep- Capturar en la pizarra los contos (5 min)

10. Preparación de la clase (10 $\mathrm{min}$ )

11. Nuevos conceptos (15 min)

12. Problema (20 min)

\section{Planteamiento de problema tipo examen (35 min)}

\author{
14. Feedback (10 \\ min)
}

15. Preparación de la clase (10 min)

\section{Planteamiento de problema tipo examen (56 min)}

\section{ceptos básicos tratados}

Saludos y breve feedback de la sesión anterior.

Preguntas en alto acerca nuevos conceptos.

Se puede consultar cualquier tipo de fuente.

Únicamente los repetidores podrán participar de forma directa sin consulta de ninguna fuente.

Tiempo para leer el problema y procesarlo. Pueden consultar cualquier tipo de fuente.

Deberían saber empezar.

Los alumnos trabajarán en grupos de 4 personas. Tendrán 15' para intentar trabajar entre ellos, el profesor se acerca para ir haciendo preguntas clave que les oriente en la resolución del ejercicio. Una vez superadas las trabas iniciales, se dejan otros 30' para resolver el problema, con la supervisión del profesor.

Puntos clave aprendidos y dudas sin resolver

Saludos y breve feedback de la sesión anterior.

Los alumnos trabajarán en grupos de unas 4 personas. Los problemas de examen son 90', con lo que proporcionalmente a los puntos, tienen $36^{\prime}$. La finalidad es que se vayan acostumbrando al ritmo de examen. La resolución se hará en otros 20', con la supervisión del profesor.
Curva de enfriamiento Regla de las fases de Gibbs

Equilibrio

Tipos de diagramas

Transformación peritéctica Curva de enfriamiento
Introducción al inequilibrio 
Algunos fenómenos de inequilibrio: Deformación de las líneas del diagrama, Porosidad, Quemado, Microsegregación interdendrítica, coring dudas al respecto o
aclaración de con-
ceptos (10 min)

19. Feedback ( 4 20. Preparación de clase (10 min)

\section{Mini problemas}


27. Planteamiento de problema tipo examen (36 min)

Los alumnos trabajarán en grupos de unas 4 personas. Los problemas de examen son 90', y un diagrama vale 4 puntos, con lo que proporcionalmente tienen 36'. La finalidad es que se vayan acostumbrando al ritmo de examen. La resolución se hará sobre la marcha en la pizarra. Tienen que resolver el problema con la supervisión del profesor.

\section{Repetición cuestionario (20min)}

\section{Feedback (6 min) \\ Despedir el tema y feedback de lo aprendido.}

Como se observa en el listado de tareas, se empieza y se termina el ciclo con una actividad común: un cuestionario. Éste se emplea al comienzo del CIMA para conocer el nivel del que parten los alumnos. Dicho cuestionario se repite al finalizar el ciclo, sirviendo su análisis al profesor como herramienta para conocer dónde se encuentran las dificultades en el aprendizaje del tema, y poder mejorar así la docencia en futuras aplicaciones de este CIMA del tema de Diagramas en cursos venideros. Se les da a los alumnos la opción de entregarlo con su nombre real o bien usar un pseudónimo, recordándoles en todo caso, que tiene que poder establecerse una relación entre los cuestionarios inicial y final.

El cuestionario debe redactarse en lenguaje coloquial, principalmente por la escasez de conocimientos de la que parten. En este CIMA se incorporaron un total de 8 preguntas:

1. Describe con tus propias palabras lo que crees que puede ser las lineas de LIQUIDUS, SOLIDUS y SOLVUS. 
2. Los cambios de fase en los materiales, ¿se dan de forma instantánea? Si estamos enfriando una aleación, ¿qué crees que ocurrirá cuando se esté dando esa transformación espontánea en el material?

3. ¿Se podría conocer la microestructura de un material, bajo unas determinadas condiciones de composición y temperatura, a partir de un diagrama de fases? Explica aproximadamente cómo se conocería.

4. A partir de un diagrama de equilibrio, ¿se podría conocer la evolución de la microestructura con la temperatura?

5. Nombra los tratamientos térmicos que conozcas; se someten los materiales a cambios de temperatura para modificar sus propiedades finales. No hace falta que describas dichos cambios, aunque genial si pudieras hacerlo.

6. ¿Conoces algún fenómeno causado en los materiales por un enfriamiento más rápido de la cuenta? Nombra y describe cada uno que conozcas.

7. ¿Cómo crees que afectaría a un material el enfriarlo más rápido de la cuenta desde el estado líquido?

8. ¿Y si lo calentamos desde temperatura ambiente y luego lo enfriamos muy rápidamente?

\section{Aplicación del CIMA}

A continuación, se procede a la descripción de las sensaciones percibidas durante la implantación del ciclo de mejora, junto con la reflexión y análisis de las mismas, sin perder la vista en las futuras aplicaciones a desarrollar del CIMA. En lugar analizar de forma general las diferentes sesiones, se ha preferido hacerlo de forma particular conforme se iban desarrollando las mismas, comentando cada actividad planteada en un principio. Se incluyen en la 
tabla 2 los avances conseguidos, el feedback de los alumnos y las dificultades encontradas en el proceso, que no han sido pocas.

Tabla 2.- Reflexiones y análisis del CIMA

\begin{tabular}{|l|l|}
\hline Tarea & Sensaciones \\
\hline $\begin{array}{l}\text { 1. Preparación de la } \\
\text { clase (5') }\end{array}$ & $\begin{array}{l}\text { Ya no les pilla tan de sorpresa como ocurrió con el } \\
\text { ciclo básico de mejora. (3 min) }\end{array}$ \\
\hline $\begin{array}{l}\text { 2. Cuestionario ini- } \\
\text { cial (20') }\end{array}$ & $\begin{array}{l}\text { vaya a evaluar; con lo que la mayoría escribe un } \\
\text { pseudónimo, y sólo unos pocos escriben su ver- } \\
\text { dadero nombre. Al insistir en que no puntúa, algu- } \\
\text { nos alumnos desconectan y se dedican a charlar } \\
\text { con sus compañeros. Cuando incido en que los } \\
\text { cuestionarios deben responderse de forma indivi- } \\
\text { dual, dos alumnos los entregan prácticamente en } \\
\text { blanco; resulta frustrante como ignoran activida- } \\
\text { des que requieren un cierto esfuerzo, si no acom- } \\
\text { paña un premio. (20 min) }\end{array}$ \\
\hline $\begin{array}{l}\text { 3. Formación de } \\
\text { grupos (5') }\end{array}$ & $\begin{array}{l}\text { Los grupos se forman más rápido que en la pri- } \\
\text { mera ocasión, ya están acostumbrados y enseguida } \\
\text { se disponen a trabajar. (3 min) }\end{array}$ \\
\hline $\begin{array}{l}\text { 4. Problema: en- } \\
\text { trega enunciado } \\
\text { (5') }\end{array}$ & $\begin{array}{l}\text { Inmediatamente descubren que no saben empezar. } \\
\text { Algunos grupos piden insistentemente que se les } \\
\text { proporcione alguna clave. La clase se activa y co- } \\
\text { mienzan a integrarse. (4 min) }\end{array}$ \\
\hline tiva (5') \\
tiva (5')
\end{tabular}

Jornadas de Formación e Innovación Docente del Profesorado | № 2 (2019) Esta obra se distribuye con la licencia Creative Commons Reconocimiento-NoComercial-SinObraDerivada Internacional (CC BY-NC-ND 4.0.) 


\begin{tabular}{|c|c|}
\hline $\begin{array}{l}\text { 8. Problema: fina- } \\
\text { lizar parte } 2 \text {, pen- } \\
\text { sar sobre pregunta } \\
3\left(20^{\prime}\right)\end{array}$ & $\begin{array}{l}\text { Hubo un aluvión de preguntas, no sólo a lo que re- } \\
\text { fiere la pregunta } 2 \text { del problema que concernía a } \\
\text { cómo realizar un análisis de fase completo, sino } \\
\text { que se adelantaron nerviosos a la pregunta } 3 \text {. ( } 20 \\
\text { min) }\end{array}$ \\
\hline $\begin{array}{l}\text { 8b. Píldora } \\
\text { informativa }\end{array}$ & $\begin{array}{l}\text { Esta actividad no estaba prevista en esta sesión. } \\
\text { Quería dejarles con sensación de suspense para } \\
\text { comenzar con gancho en la siguiente clase, pero } \\
\text { presionaron y hubo que incluir la explicación del } \\
\text { concepto de curva de enfriamiento en esta sesión. } \\
\text { (10 min) }\end{array}$ \\
\hline $\begin{array}{l}\text { 9. Resumen con- } \\
\left.\text { ceptos ( } 5^{\prime}\right)\end{array}$ & $\begin{array}{l}\text { Debido a la introducción de la nueva píldora infor- } \\
\text { mativa, no se pudo realizar el resumen de concep- } \\
\text { tos en la pizarra. }\end{array}$ \\
\hline $\begin{array}{l}\text { 10. Prepara-ción } \\
\text { clase (10') }\end{array}$ & $\begin{array}{l}\text { Después de los saludos habituales, se recupera en } \\
\text { pantalla el problema. ( } 5 \text { min) }\end{array}$ \\
\hline $\begin{array}{l}\text { 11. Nuevos concep- } \\
\text { tos (15') }\end{array}$ & $\begin{array}{l}\text { La sensación desde el comienzo de la clase es que } \\
\text { están un poco perdidos, parece como si se hubie- } \\
\text { ran olvidado por completo de la forma de trabajo } \\
\text { de la sesión anterior. No quiero hablar más de lo } \\
\text { establecido, por lo que corto para dar paso a la re- } \\
\text { solución de problemas, aplicando lo explicado en } \\
\text { ésta y en la anterior sesión. (15 min) }\end{array}$ \\
\hline 12. Problema (20') & $\begin{array}{l}\text { Continúan perdidos, de hecho, bastante atorados, } \\
\text { incluso en la redacción de ecuaciones extremada- } \\
\text { mente sencillas. Algunos llegan tarde y bromean } \\
\text { con que han borrado su memoria porque vienen } \\
\text { de otro examen. } \\
\text { El problema en cuestión es sencillo y trata simple- } \\
\text { mente de la aplicación de la regla de la palanca. } \\
\text { Se explica de nuevo, grupo a grupo, para que re- } \\
\text { cuerden cómo funciona; no quiero repetir la mini } \\
\text { lección magistral de la sesión anterior, quiero que } \\
\text { se esfuercen en pensar sobre ella. Algunos la re- } \\
\text { cuerdan, pero no saben cómo aplicarla. ( } 25 \text { min) }\end{array}$ \\
\hline
\end{tabular}

Jornadas de Formación e Innovación Docente del Profesorado | № 2 (2019) Esta obra se distribuye con la licencia Creative Commons Reconocimiento-NoComercial-SinObraDerivada Internacional (CC BY-NC-ND 4.0.) 


\begin{tabular}{|c|c|}
\hline $\begin{array}{l}\text { 13.Planteamien-to } \\
\text { de problema tipo } \\
\text { examen (35') }\end{array}$ & $\begin{array}{l}\text { El problema que se les propone en esta activi- } \\
\text { dad es similar a uno de examen, aunque simplifi- } \\
\text { cado en su enunciado, pero que les sirva para irse } \\
\text { acostumbrando al uso del lenguaje técnico carac- } \\
\text { terístico del tema. A pesar de ser más simple, hoy } \\
\text { no tienen buen aspecto, se les ve agotados. Ni si- } \\
\text { quiera los alumnos más avanzados, hoy están rin- } \\
\text { diendo; intento distender el ambiente con alguna } \\
\text { broma. Poco a poco avanzan en la resolución, pero } \\
\text { necesitan más tiempo del previsto. ( } 45 \text { min) }\end{array}$ \\
\hline 14. Feedback (10') & $\begin{array}{l}\text { Piden que se les proporcione alguna colección de } \\
\text { problemas resueltos. Les argumento que no les } \\
\text { conviene, puesto que entonces no entenderían la } \\
\text { asignatura del todo. Me dan la razón, porque tie- } \\
\text { nen claro que no estudian hasta el último minuto. } \\
\text { Les recuerdo que en la bibliografía se les cita un li- } \\
\text { bro de problemas en el que están muy bien expli- } \\
\text { cados los diagramas, y lo agradecen. Se prolonga la } \\
\text { charla hasta que se apaga la luz del aula. } \\
\text { Sinceramente, estoy muy contenta porque, aunque } \\
\text { hoy ha sido un día duro para ellos, se respira otro } \\
\text { ambiente en la clase. (10 min) }\end{array}$ \\
\hline $\begin{array}{l}\text { 15. Prepara-ción de } \\
\text { la clase (10') }\end{array}$ & $\begin{array}{l}\text { No reaccionan bien al saber que se va a hacer un } \\
\text { problema real de examen con tiempo real de exa- } \\
\text { men; preguntan si se les evaluará de alguna forma. } \\
\text { Al saber que no, se relajan bastante, pero no pier- } \\
\text { den motivación. (10 min) }\end{array}$ \\
\hline
\end{tabular}

Jornadas de Formación e Innovación Docente del Profesorado | № 2 (2019) Esta obra se distribuye con la licencia Creative Commons 
16. Plantea-miento de problema tipo examen (56')
Muchos se ponen a copiar el enunciado y no puedo evitar indignarme, porque lo tienen accesible online por varias vías. Inicialmente no entendía por qué perdían tiempo, pero rápidamente comprendí que se limitaban a retrasar el enfrentarse al problema, y así, no salir a la pizarra.

Varios recuerdan la recomendación del libro de problemas, y me piden poder ir a la biblioteca a sacarlo. Me encanta la propuesta porque presupone que tienen ganas de resolver el problema. Por supuesto que pueden contar con todas las herramientas que deseen para afrontar la actividad, con lo que de repente tengo a unos 10 alumnos que salen corriendo de la clase.

De vuelta y resituados, con todas las herramientas que creen necesarias, muchos están como empezando desde cero. Voy ayudando, aunque me frustro cuando compruebo que es cierto que no se acuerdan de nada. Aprecio, como un rayo de esperanza, que a poco que se les cuente, enseguida saben continuar... así que me calmo y sigo ayudándoles con el atasco.

Decido dar 45' antes de sacar a nadie a resolverlo, con la posibilidad de que pueden seguir con el problema mientras se va mostrando la resolución en la pizarra. (70 min)

17. Nuevos concep$\operatorname{tos}\left(\mathbf{1 0}^{\prime}\right)$

Se cumple con el tiempo estipulado. (10 min) 
18. Resolución de dudas al respecto 0 aclaración de conceptos (10')
Las dudas se fueron resolviendo durante el ejercicio, siempre intentando que ellos mismos las explicasen a sus compañeros.

De inequilibrio no ha surgido ninguna duda, por lo que no se han podido enterar de mucho. Deduzco que estaban emocionados porque, poco a poco, les iba saliendo el problema y no querían desconcentrarse en algo que se estuviera explicando en la pizarra. Mis subidas al estrado ahora, en su práctica mayoría, son para explicar algo a un determinado grupo, con más sencillez, pero aprovechando las transparencias. Por tanto, aun cuando se les avisa de que por favor atiendan, no ven los contenidos como lecciones magistrales. Así que tanto si están concentrados en la resolución de un problema, como si están discutiendo entre ellos cómo resolverlo, suelen ignorar las subidas a la pizarra. Solamente cuando les atañe directamente o bien porque estén atascados esperando a que me quede libre, están atentos escuchándome. (0 min)

Se acorta lo posible, ya que integramente esta actividad tiene que hacerse fuera del tiempo establecido de clase. Pero insisto en hacer la actividad, puesto que no quiero que me pase como en la sesión anterior y no poder hacer el resumen de conceptos aprendidos en la sesión. (3 min)

Después de los saludos, les pregunto sobre el inequilibrio, por la sensación de la sesión anterior,

20. Prepara-ción de la clase (10') intuía que no lo habían entendido. Efectivamente, no sólo no se enteraron, sino que algunos ni me escucharon al estar concentrados en los problemas. Se rehace sobre la marcha el plan de actividades y se enfoca de otra forma. (15 min) 


\begin{tabular}{|c|c|}
\hline $\begin{array}{l}\text { 21. Mini problemas } \\
(25)\end{array}$ & $\begin{array}{l}\text { Ya no tiene sentido ponerles a trabajar en peque- } \\
\text { ños grupos y asignarles mini problemas de inequi- } \\
\text { librio. Además, cada grupo iba a tener diferentes } \\
\text { problemas, con lo que, si alguno se despistara, po- } \\
\text { dría interpretar incorrectamente mi explicación a } \\
\text { otro grupo. Se decide pues resolver los mini pro- } \\
\text { blemas entre todos y eliminar el reparto a los } \\
\text { grupos. } \\
\text { Resulta curioso cómo siguen trabajando con los } \\
\text { grupos inicialmente establecidos, les hace sentir } \\
\text { más cómodos el no afrontar un problema de forma } \\
\text { individual. Hay que alargar el tiempo establecido } \\
\text { para poder llegar a } 6 \text { de los } 8 \text { problemas previstos. } \\
\text { (35 min) }\end{array}$ \\
\hline $\begin{array}{l}\text { 22. Nuevos concep- } \\
\text { tos (10') }\end{array}$ & $\begin{array}{l}\text { Se cumple con el tiempo estipulado para el desa- } \\
\text { rrollo de la actividad. (10 min) }\end{array}$ \\
\hline $\begin{array}{l}\text { 23. Preguntas tipo } \\
\text { test (15') }\end{array}$ & $\begin{array}{l}\text { Se mantienen estas dos actividades para no salir- } \\
\text { nos demasiado del esquema inicial. ( } 15 \mathrm{~min} \text { ) }\end{array}$ \\
\hline $\begin{array}{l}\text { 24a. Propuesta } \\
\text { problema examen } \\
\left(30^{\prime}\right)\end{array}$ & $\begin{array}{l}\text { Al partirse el esquema propuesto para la clase, no } \\
\text { hay armas para engancharles con el problema que } \\
\text { se propone; 15' no bastan para meterse de lleno en } \\
\text { el problema y que lo continúen en casa. De todas } \\
\text { formas, insisto que sigan en casa, y que se reco- } \\
\text { gerá el próximo día en clase. ( } 15 \text { min) }\end{array}$ \\
\hline $\begin{array}{l}\text { 24b. Resumen } \\
\text { conceptos }\end{array}$ & $\begin{array}{l}\text { Para no quedarme con la sensación de perder- } \\
\text { los del todo, me empeño en dar un pequeño re- } \\
\text { sumen en la pizarra con los conceptos impartidos, } \\
\text { incluyendo un esquema de las diferentes microes- } \\
\text { tructuras obtenidas en inequilibrio; se hace con la } \\
\text { ayuda de algunos alumnos. Aviso que es recomen- } \\
\text { dable y voluntario, pero que, si se tienen que ir, va- } \\
\text { yan abandonando la clase silenciosamente. Un } \\
\text { tercio de los alumnos abandonan la clase, otro ter- } \\
\text { cio participa activamente en la elaboración del re- } \\
\text { sumen y el tercio restante va recogiendo a la vez } \\
\text { que escucha lo que se va comentando. ( } 4 \text { min) }\end{array}$ \\
\hline $\begin{array}{l}\text { 25. Preparación de } \\
\text { la clase (8') }\end{array}$ & $\begin{array}{l}\text { Después de los habituales saludos y prepara- } \\
\text { ción de la clase en el ordenador, se avisa que se } \\
\text { va a recoger el problema propuesto en clase el úl- } \\
\text { timo día, pero nadie lo ha traído resuelto. Por el in- } \\
\text { cumplimiento de la tarea, pierden la gratificación } \\
\text { prometida, 0,1 puntos por salir a resolverlo a la pi- } \\
\text { zarra. (8 min) }\end{array}$ \\
\hline
\end{tabular}

Jornadas de Formación e Innovación Docente del Profesorado | № 2 (2019) Esta obra se distribuye con la licencia Creative Commons Reconocimiento-NoComercial-SinObraDerivada Internacional (CC BY-NC-ND 4.0.) 


\begin{tabular}{|l|l|}
\hline $\begin{array}{l}\text { 26. Problema en pi- } \\
\text { zarra (20') }\end{array}$ & $\begin{array}{l}\text { Se dejan 10' para retomar la resolución del pro- } \\
\text { blema y comienzan a salir alumnos a la pizarra. (50 } \\
\text { min) }\end{array}$ \\
\hline $\begin{array}{l}\text { 27. Plantea-miento } \\
\text { de problema tipo } \\
\text { examen (36') }\end{array}$ & $\begin{array}{l}\text { Hay que cancelar esta actividad para poder cen- } \\
\text { trarse en el anterior problema. (0 min) }\end{array}$ \\
\hline $\begin{array}{l}\text { 28. Repetición } \\
\text { cuestionario inicial } \\
\text { (20') }\end{array}$ & $\begin{array}{l}\text { Les cuesta mucho ponerse con el cuestionario. En } \\
\text { un par de casos, al no haber estado en la primera } \\
\text { sesión y no haber hecho el cuestionario inicial, in- } \\
\text { tentan no hacer el final. Los más activos por lo } \\
\text { cuperar la décima perdida de la participación en } \\
\text { la resolución del problema, proponiendo ganar } \\
\text { esa décima por el esfuerzo que supone realizar el } \\
\text { cuestionario. También se hace caso omiso de esta } \\
\text { petición, aunque resultan muy divertidos los inten- } \\
\text { tos de embaucamiento. (25 min) }\end{array}$ \\
\hline $\begin{array}{l}\text { 30. Tutoría } \\
\text { colectiva }\end{array}$ & $\begin{array}{l}\text { Dejo que termine la clase unos minutos antes de } \\
\text { la hora. No quiero que piensen que tener un CIMA } \\
\text { suponga alargar las clases. (4 min) }\end{array}$ \\
\hline $\begin{array}{l}\text { Tras corregir casi la mitad de los cuestionarios, se } \\
\text { decide alargar el Cima en una sesión más de lo ini- } \\
\text { cialmente previsto. Los alumnos seguían sin tener } \\
\text { claros los conceptos de inequilibrio. } \\
\text { La mitad de la clase se empleó en incidir sobre di- } \\
\text { chos conceptos en forma de lección magistral, } \\
\text { mientras que la otra mitad de la sesión sirvió para } \\
\text { resolver dudas que les habian surgido a aquellos } \\
\text { que ya habian avanzado en el estudio del tema. } \\
\text { (60 min) }\end{array}$ \\
\hline (6)
\end{tabular}

En general la respuesta de los alumnos al CIMA ha sido muy buena. Ha mejorado tanto la comunicación profesor-alumno, como su actitud en clase. Destaca en este cambio metodológico el hecho de que el profesor se convierte en otra herramienta más de aprendizaje, mientras que los alumnos adquieren un papel protagonista.

A continuación, se presentan los resultados de las escaleras de aprendizaje, es decir, el análisis de los cuestionarios. Se hará un análisis pormenorizado de cada 
pregunta, para pasar después a un análisis por individuo, pero con una evaluación global del cuestionario, poniéndole nota. Para estudiar la evolución global, se ha presupuesto a cada una de las categorías una puntuación del 1 al 5, desde la categoría E hasta la A, obteniéndose la última gráfica de la figura 3.

La gran dificultad que se ha encontrado en este análisis reside en la reflexión sobre qué causa cada escalón en el aprendizaje. No es una tarea sencilla, ya que marca los obstáculos que tienen los alumnos al ir entendiendo la asignatura.

Por lo general, la evolución ha sido bastante buena en la clase. En el estudio global por estudiante destaca el hecho de que uno de los alumnos bajara en su calificación debido, probablemente, al cansancio contestando el cuestionario final; habían tenido examen esa mañana, la clase era a las 15:30 en un aula regularmente acondicionada para las altas temperaturas.
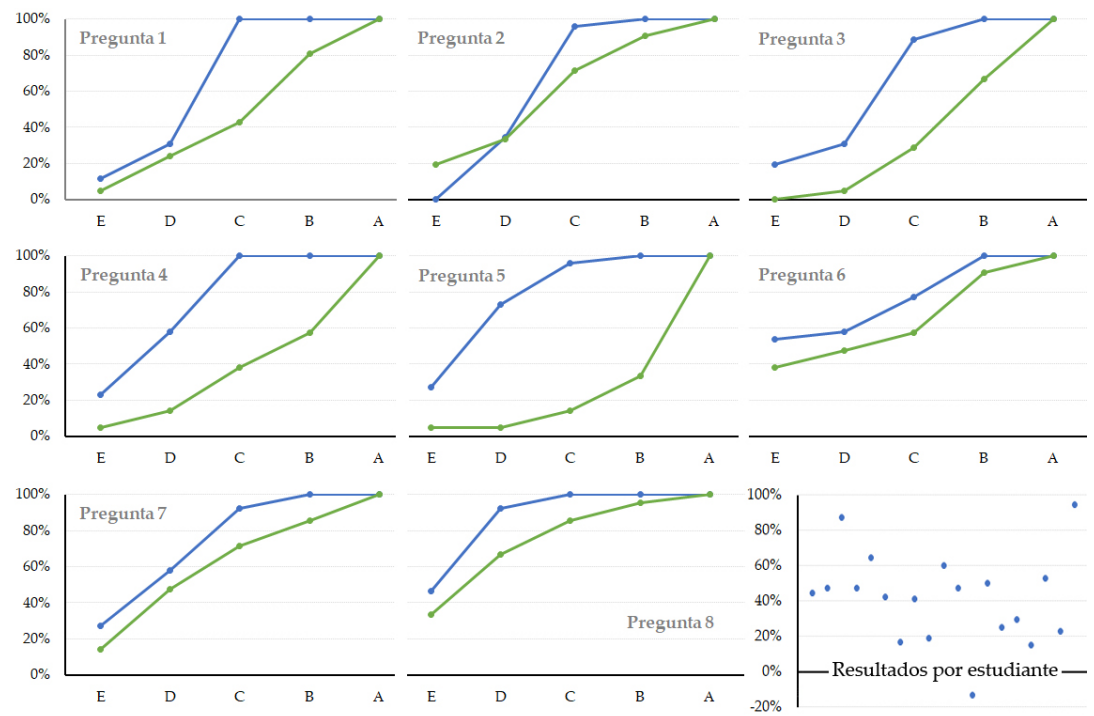

Figura 3. Escaleras de aprendizaje

Jornadas de Formación e Innovación Docente del Profesorado | № 2 (2019) Esta obra se distribuye con la licencia Creative Commons Reconocimiento-NoComercial-SinObraDerivada 
Ha habido dos principales obstáculos encontrados en la comprensión del tema, que han sido las transformaciones de fase y el inequilibrio, con toda la casuística que lo rodea. Esto exige una seria reformulación en la forma en la que se imparten actualmente estos conceptos dentro del CIMA, para lograr vencer la barrera que en la actualidad suponen.

\section{Evaluación del CIMA}

De todo lo anteriormente visto, se trasluce una ligera reconfiguración del ciclo para su futura implantación en cursos venideros. De forma general, se mantendrá esta forma de impartir el temario en este tema, planteándose la integración de esta metodología poco a poco en otros más. Se centrará la docencia en la resolución de problemas, limitando los conocimientos conceptuales a dosificadas píldoras informativas que se centrarán en los conceptos clave para la continuación de la resolución del ejercicio y evitar el estancamiento del alumnado. En particular, debe hacerse una nueva reestructuración a los apartados que han supuesto un hándicap para los alumnos, como han sido los conceptos de transformaciones de fase, e inequilibrio.

Debe cuidarse la redacción de los cuestionarios que correspondan a los temas nuevos a incorporar al ciclo de mejora. Se propone incluso la realización de alguna prueba eligiendo algún estudiante, preferentemente repetidor, para poder estudiarlo como si fuera el cuestionario final en su caso. Habría que sacrificar dicho resultado para el estudio de la evolución del grupo, pero serviría de gran ayuda para comprobar si el resto de los compañeros podrían ser capaces de comprender correctamente lo que se les pregunta.

Se destaca la necesidad de contar con actividades adicionales, para que cuando el desarrollo de la clase rompa 
el esquema inicial planteado en la sesión, no deje coja la clase y se pueda seguir avanzando con el tema en cuestión, sin perder el interés de los estudiantes.

La implementación del ciclo ha sido dura pero satisfactoria. Superado el entusiasmo inicial de los estudiantes, se hizo bastante dificil mantener la atención, implicación y entrega de los estudiantes, necesarias en esta metodología. Me siento feliz por haber sido capaz de llegar, al menos, a una parte del alumnado y me propongo, con fuerza, seguir llegando cada curso a más si fuera posible.

Se empieza el CIMA con una idea, pero se termina con otra mucho mejor. El comienzo es ilusionante, parece sencillo, una simple cuestión de tiempo para preparar el material docente a impartir. Pero conforme se avanza, se revuelven los esquemas internos de docencia, y la metodología cobra vida en el aula.

Puede que lo más importante que haya aprendido en la aplicación de este CIMA haya sido que hay que irse enfrentando a pequeños objetivos; cuando se quiere abarcar mucho, es normal fallar entre tantas labores investigadoras y docentes. Puedes rendirte y no lograr conectar ni con los estudiantes, ni con uno mismo. No vale pensar como ellos, ni empatizar con ellos: hay que retarles a un nuevo aprendizaje. Aquel en el que igual no cuentan con todos los conceptos (por eso de la selección y priorización de contenidos para poder cuadrarlo con el calendario académico), pero van a aprender a PENSAR. Los alumnos se convierten así en el centro de la clase; los contenidos no son ya lo importante, crecen con ellos. Los alumnos se convierten, de alguna manera, en profesores.

En cuanto a los contenidos a impartir, destaco lo revelador que ha sido en mi caso realizar un mapa de contenidos. Su elaboración supone un enorme reto, porque en el fondo, se sigue siendo reacio al cambio. Con el mapa, al 
tener el tema en una sola imagen, se establecen casi sin querer las bases y las relaciones para poder entenderlo.

Con el CIMA he podido comprobar que ciertas metodologías que ya aplicaba son de gran ayuda y que, de hecho, están respaldadas por excelentes profesores. El tema de las preguntas clave es una de ellas, aunque he mejorado en su aplicación. Antes era yo la que preguntaba y respondía, mientras que ahora son ellos los que tienen que buscar las repuestas. Ahora están realmente involucrados en su proceso de aprendizaje.

El cambiar el modelo metodológico hace sentirse más inseguro en las clases, pero a la vez se conecta más con los alumnos porque todos estamos en un proceso de aprendizaje. El profesor debe encontrar los obstáculos que frenan ese camino: si se les ayuda a superarlos, ellos avanzan más.

También he descubierto algo obvio, que algo nuevo sorprende y gusta, pero que si se repite deja de causar esa fascinación. Hay que cuidar la secuencia de actividades y procurar que sea diversa para poder mantener la atención de todo el alumnado, y no sólo la de los pocos que habitualmente ya atendían.

Con todo ello, me dispongo a cambiar mi metodología, como mínimo, a tema por año. En general estoy muy contenta con los resultados, muy motivada para vencer el absentismo en próximas convocatorias. 


\section{Referencias bibliográficas}

Bain, K. (2007). Lo que hacen los mejores profesores universitarios. Valencia: Universidad de Valencia.

Cano, E. y Barrios, R. (2011). Buenas prácticas en la evaluación de competencias: cinco casos de Educación Superior. Barcelona: Laertes.

Escribano, A. y Del Valle, A. (2008). El aprendizaje basado en problemas: Una propuesta metodológica en Educación Superior. Madrid: Narcea.

Finkel, D. (2008). Dar clase con la boca cerrada. Valencia: Universidad de Valencia.

Harland, T. (2017). Enseñanza universitaria: Una guía introductoria. Madrid: Morata.

Porlán, R. (Coord.) (2017). Enseñanza universitaria: Cómo mejorarla. Madrid: Morata.

Jornadas de Formación e Innovación Docente del Profesorado | № 2 (2019) Esta obra se distribuye con la licencia Creative Commons Reconocimiento-NoComercial-SinObraDerivada 4.0 Internacional (CC BY-NC-ND 4.0.) 